\title{
HIGH MASS FLUX MASS TRANSFER THROUGH A MOBILE INTERFACE
}

\author{
AKIRA HIRATA ANd Yoshizo SUZUKI \\ Chemical Engineering Course, Department of \\ Applied Chemistry, Waseda University, Tokyo 160
}

\begin{abstract}
A theoretical study was made of mass transfer in a laminar boundary layer through a mobile interface with high mass flux condition. The laminar boundary momentum and diffusion equations are solved numerically with Blasius-type similar transformations. The numerical results are obtained for mass transfer rates by taking account of the diffusional flow due to rapid mass transfer and interfacial velocity due to motion of interface simultaneously. Mass transfer rates are given as function of transfer number $B$ (a dimensionless driving force of mass transfer to be related to diffusional flow), interfacial velocity parameter $\boldsymbol{u}_{s} / \boldsymbol{u}_{\infty}$ and Schmidt number. The results show that the interfacial velocity increases the mass transfer rates in the case of high mass flux as well as for Iow mass flux. This effect is very important in the high-Schmidt number region. The previous theories of high mass flux phenomena with fixed interface can be applied to high mass flux mass transfer with mobile interface as long as the effect of the interfacial velocity, in increasing the mass transfer rates, is considered.
\end{abstract}

\section{Introduction}

It is very important to take account of the effects of the interfacial velocity, which is caused by the tangential motion of an interface like a falling liquid film, for prediction of the rates of momentum, heat and mass transfer through a heterogeneous interface. Acrivos $^{1)}$ analyzed the steady laminar gas phase mass transfer rates on falling liquid film by using the laminar boundary layer theory in the case of Schmidt number of near unity. Hirata ${ }^{13,14,19)}$ extended the study of Acrivos for a wide range of Schmidt number and pointed out several effects of the interfacial velocity on mass transfer rates. One of the most important effects is the increase of mass transfer rates, especially in liquid-phase mass transfer.

The purpose of this type of research is to make clear the effects of tangential velocity at the interface on mass transfer rates. On the other hand, there is a field of high mass flux mass transfer to make clear the effects of normal velocity at the interface on mass transfer rates. For high mass flux mass transfer, the effects of convective velocity, which is caused by rapid mass transfer and is normal to the interface, on the velocity field near the interface cannot be neglected. The mass transfer rate itself is influenced by it. A number of studies on high mass flux phenomena with fixed interface have been made, most of them analyses by boundary layer theory. Useful reviews were

\footnotetext{
Received August 7, 1979. Correspondence concerning this article should be addressed to A. Hirata. Y. Suzuki is now at Nat1. Res. Inst. for Pollution and Resources, Tsukuba 305.
}

given by $\mathrm{Asano}^{3)}$ and Nienow ${ }^{17)}$. Results were shown for the effects of transfer number $B$, a dimensionless driving force for mass transfer, instead of the diffusional velocity itself. It is determined that the mass transfer rate is highly influenced by dimensionless driving force ${ }^{20,22)}$

The studies on these two fields mentioned above were done independently. Therefore, there are few studies taking account of the interfacial velocity in high mass flux mass transfer ${ }^{6}$, though the effects of interfacial velocity on mass transfer rates have been emphasized in the case of low mass flux. Clark and King $^{7}$ suggested the effects of interfacial velocity on mass transfer rates under high mass flux conditions. However, they did not refer to its quantitative effects on mass transfer rates. In actual equipment, high mass flux mass transfer with interfacial velocity must occur. Therefore, it is of interest to take account of interfacial velocity in high mass flux phenomena. The authors ${ }^{157}$ analyzed the momentum and heat transfer through a moving flat plate within a constant free stream with forced injection or suction. In this paper the case in which injection or suction velocities are caused by rapid mass transfer is presented.

\section{Fundamental Equations}

In an attempt to arrive at a better understanding, a simple model is set up for high mass flux mass transfer with mobile interface as shown in Fig. 1. Consider a system of cocurrent parallel flows, phases I and II, and a transfer of single component A through phase I. (Phase II need not be fluid; it can be a moving flat 
plate at constant velocity $u_{s}$ within a constant free stream.) We assume that mass transfer resistance exists only in phase I, and the mass transfer rate is so large (high mass flux mass transfer) that the effects of the normal velocity component at the interface, $v_{s}$, caused by mass transfer cannot be neglected. For example, the evaporation of volatile pure liquids, the condensation of vapors with the noncondensable gas and the dissolution of highly soluble liquids can be considered. For steady two-dimensional laminar flow with uniform fluid properties, the transport equations for phase I are written as follows:

$$
\begin{aligned}
& \partial u / \partial x+\partial v / \partial y=0 \\
& u(\partial u / \partial x)+v(\partial u / \partial y)=\nu\left(\partial^{2} u / \partial y^{2}\right) \\
& u(\partial w / \partial x)+v(\partial w / \partial y)=\mathscr{D}\left(\partial^{2} w / \partial y^{2}\right)
\end{aligned}
$$

Boundary conditions are

$$
\begin{aligned}
& y=0: u=u_{s}(=\text { const }) \\
& v=v_{s}(x)=-\left.\frac{\mathscr{D}}{1-w_{s}} \frac{\partial w}{\partial y}\right|_{y=0} \\
& w=w_{s}(=\mathrm{const}) \\
& y=\infty: u=u_{\infty}(=\mathrm{const}), w=w_{\infty}(=\mathrm{const})
\end{aligned}
$$

The condition of $v_{s}$ in Eq. (4b) is derived by the condition of the uni-directional diffusion of component $\mathrm{A}$ at the interface. The interfacial velocity, $u_{s}$, would be determined by the shearing stress balance at the interface for a fluid-fluid system ${ }^{24}$. But here, the interfacial velocity, $u_{s}$, is assumed to be a given, constant value along the interface in order to simplify the problem. Equations (1)-(3) are rewritten by introducing the well known Blasius-type similar transformations as follow:

$$
\begin{aligned}
& \eta=(y / x) \operatorname{Re}_{x}{ }^{1 / 2} \\
& \phi=\left(\nu u_{\infty} x\right)^{1 / 2} f(\eta) \quad(u=\partial \psi / \partial y, v=-\partial \psi / \partial x) \\
& \phi=\left(w-w_{\infty}\right) /\left(w_{s}-w_{\infty}\right)
\end{aligned}
$$

Reduced equations and boundary conditions are

$$
\begin{aligned}
& f^{\prime \prime \prime}+\frac{1}{2} f f^{\prime \prime}=0 \\
& \phi^{\prime \prime}+\frac{1}{2} S c f \phi^{\prime}=0 \\
& f(0)=2(B / S c) \phi^{\prime}(0)=-2\left(v_{s} / u_{\infty}\right) R e_{x}^{1 / 2} \\
& f^{\prime}(0)=u_{s} / u_{\infty}, \quad \phi(0)=1 \\
& f^{\prime}(\infty)=1, \quad \phi(\infty)=0
\end{aligned}
$$

where (') denotes differentiation with respect to $\eta$ and $B$ is transfer number, a dimensionless driving force for mass transfer, defined as follows:

$$
B=\left(w_{s}-w_{\infty}\right) /\left(1-w_{s}\right)
$$

where $B>0$ implies that the diffusional velocity is toward the bulk as in the case of evaporation, while $B<0$ indicates diffusion from the bulk toward the interface as condensation. Transfer number presents the effects of the diffusional velocity.

Equation (7) cannot be solved analytically, but is

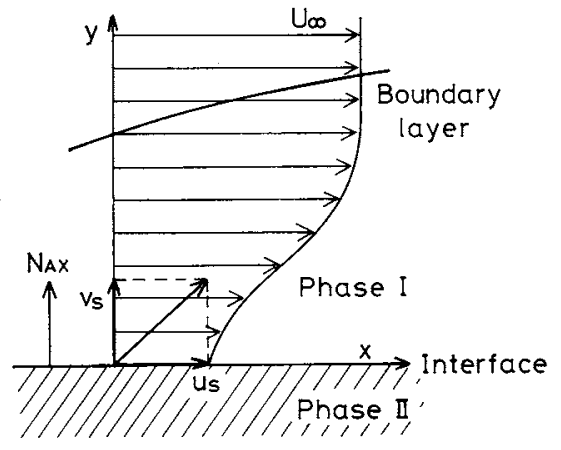

Fig. 1 Physical model of high mass flux mass transfer with a moving interface

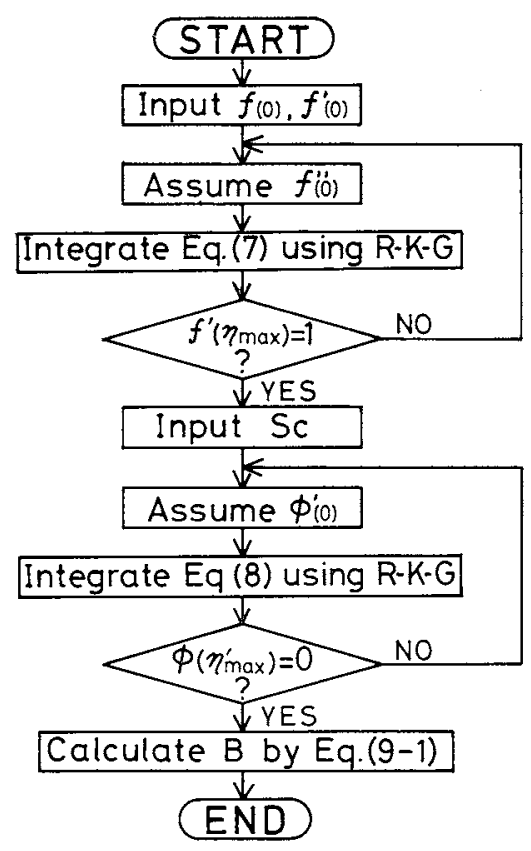

(R-K-G denotes Runge-Kutta-Gill method.)

Fig. 2 Flow chart of calculations

easily solved numerically by transforming a twopoint boundary-value problem to an initial-value problem. The flow chart of the calculations is shown as Fig. 2. Equations (7) and (8) were integrated numerically using the fourth-order Runge-KuttaGill method. The calculations were carried out for $u_{s} / u_{\infty}=0-1.0, B=-0.97-50$ and $S c=0.5,0.7,1,2$, $4,10,40,100,400,1000$ and 5000. All calculations were carried out to double-digit precision.

\section{Results and Discussion}

From Eqs. (7)-(10), we get the following fundamental relations for momentum and mass transfer.

$$
\begin{aligned}
& \left(C_{f_{x}} / 2\right) R e_{x}^{1 / 2}=\left|f^{\prime \prime}(0)\right|=F\left(u_{s} / u_{\infty}, B, S c\right) \\
& \left(1-w_{s}\right) S h_{x} R e_{x}^{-1 / 2}=-\phi^{\prime}(0) \\
& =\left\{\int_{0}^{\infty} \exp \left(-\frac{S c}{2} \int_{0}^{\eta} f d \eta\right) d \eta\right\}^{-1} \\
& =G\left(u_{s} / u_{\infty}, B, S c\right)
\end{aligned}
$$




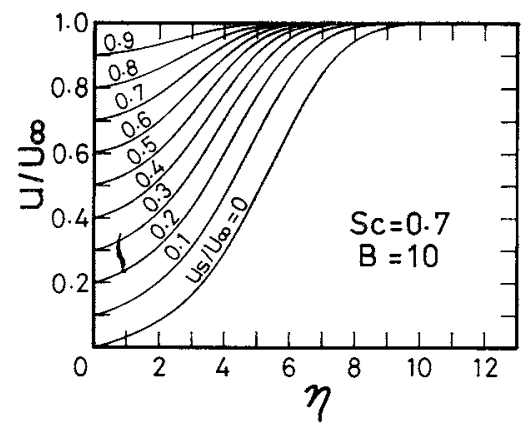

Fig. 3 Velocity profiles in boundary layer

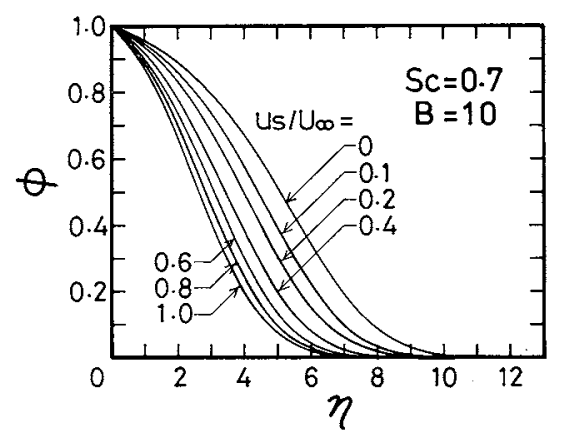

Fig. 4 Concentration profiles in diffusional boundary layer

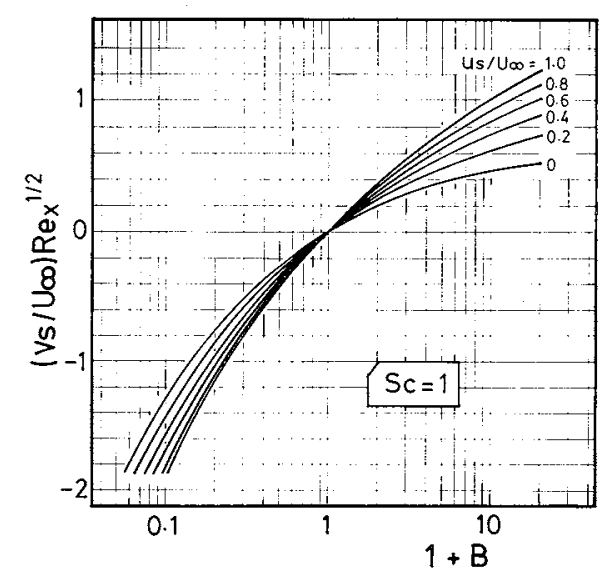

(a) Case of Schmidt number $=1$

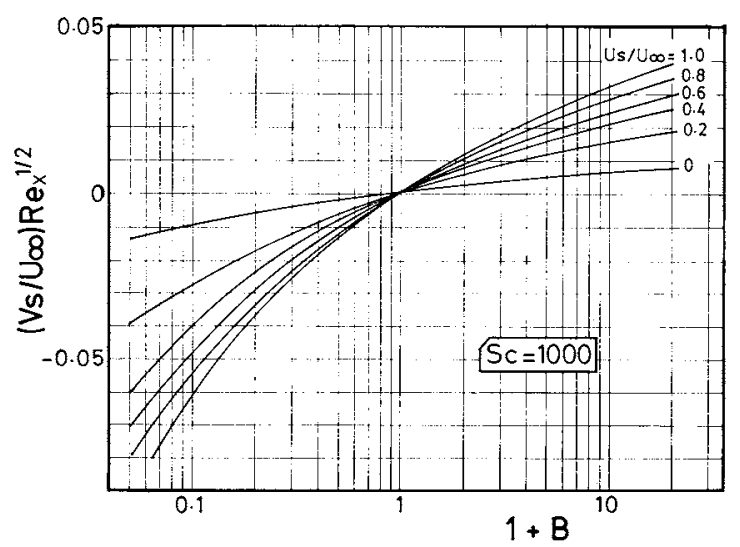

(b) Case of Schmidt number $=1000$

Fig. 5 Diffusional velocity caused by mass transfer

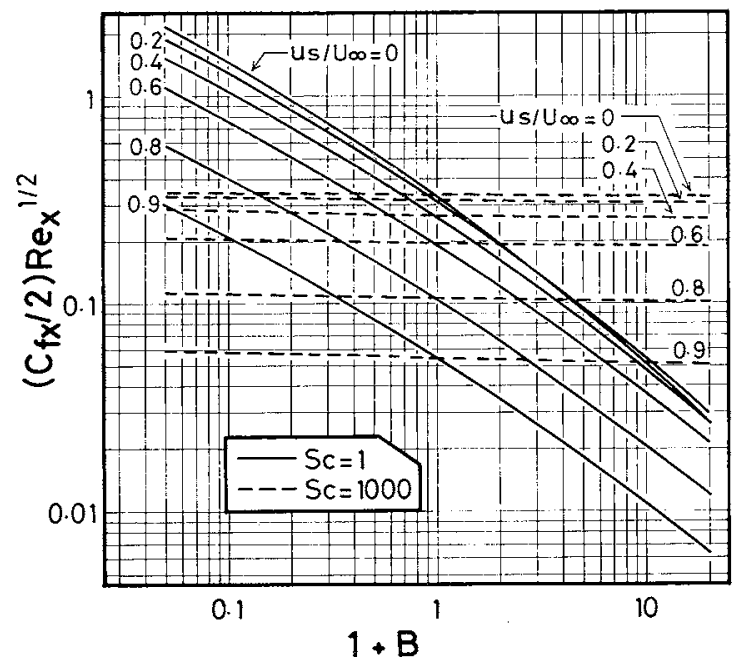

Fig. 6 Relationship between skin-friction coefficient and transfer number

where

$$
\begin{aligned}
& C_{f_{x}}=\left|\tau_{s}\right| /\left(\rho u_{\infty}{ }^{2} / 2\right) \\
& S h_{x}=k x / \mathscr{D} \\
& k=N_{A_{x}} / \rho\left(w_{s}-w_{\infty}\right)
\end{aligned}
$$

Both skin-friction coefficient and Sherwood number are functions of the interfacial velocity parameter, $u_{s} / u_{\infty}$, transfer number and Schmidt number.

\section{1 Velocity and concentration profiles}

Velocity and concentration profiles are shown as functions of $\eta$ in Figs. 3 and 4 for the case, $S c=0.7$ and $B=10$. The boundary layer flow along an interface has S-shaped velocity profiles due to rapid mass transfer toward the bulk in this condition. However, the interfacial velocity has an effect of decreasing the boundary layer thickness. Concentration profiles also have S-shaped profiles as do the velocity profiles. The interfacial velocity decreases the diffusional boundary layer thickness and increases the concentration gradient at the interface. Therefore, the mass transfer rates increase. In the case of $B<0$, the velocity and diffusional boundary layer are compressed by suction effect due to mass transfer toward the interface. However, the effect of the interfacial velocity in decreasing the velocity and diffusional boundary layer thickness is also effective.

The diffusional velocities due to mass transfer are shown in Fig. 5 for the case, $S c=1$ and 1000. From Eq. $(9 \mathrm{a}), v_{s}(x)$ satisfies the following relation.

$$
v_{s}(x) \propto \operatorname{Re}_{x}{ }^{-1 / 2}
$$

The larger value of $u_{s} / u_{\infty}$ gives larger $\left(\left|v_{s}\right| / u_{\infty}\right) R e_{x}^{1 / 2}$ at the same value of $B$. However, the order of magnitude of $v_{s}$ differs with Schmidt number (see Figs. 5 (a) and $5(\mathrm{~b}))$. The diffusional velocity, $v_{s}$, becomes small with increasing Schmidt number. Figure 6 shows the relationship between the skin-friction coef- 
ficient $C_{f_{x}},\left|f^{\prime \prime}(0)\right|$ in Eq. (12), and transfer number $B$. The skin-friction coefficient is highly influenced not only by $u_{s} / u_{\infty}$ but also by $B$ in the case of low Schmidt number. Figure 6 shows that the condition of $u_{s}=$ 0 does not give the maximum friction at sufficiently large $B^{15)}$. On the other hand, the skin-friction coefficient has nearly constant value against transfer number in the high Schmidt number region as shown in Fig. 6, and depends on $u_{s} / u_{\infty}$ only. One of the authors $^{14)}$ presented the following approximate expression for the skin-friction coefficient in the case of $B=0$ from the numerical results.

$$
\frac{C_{f_{x}}}{C_{f_{x}}{ }^{*}}=\left|1-u_{s} / u_{\infty}\right|\left\{1+(2.88-a)\left(u_{s} / u_{\infty}\right)\right\}^{1 / 2}
$$

where

$$
\begin{gathered}
a=\left\{0.09+\left(u_{s} / u_{\infty}\right)^{2}\right\} /\left\{0.1+\left(u_{s} / u_{\infty}\right)^{2}\right\} \\
C_{f_{x}}{ }^{*}=0.664 R e_{x}{ }^{-1 / 2}
\end{gathered}
$$

Equation (18a) is applicable not only to the case, $0<u_{s} / u_{\infty}<1$, but also to $u_{s} / u_{\infty}>1$, and the error does not exceed $\pm 1 \%$. Ueyama et al. ${ }^{24)}$ presented a similar approximate expression.

\subsection{Effect of transfer number on mass transfer rates}

The effect of transfer number on mass transfer rates is shown in Fig. 7. Figure 7 shows that the mass transfer rate is highly influenced by transfer number. However, it also shows that this effect is relatively independent of the interfacial velocities and Schmidt numbers. When $u_{s} / u_{\infty}=1$, the following analytical solution is obtained ${ }^{5,23)}$.

$$
\begin{aligned}
& \left(1-w_{s}\right) S h_{x} R e_{x}^{-1 / 2}=(S c / \pi)^{1 / 2} g(B) \\
& g(B)=\exp \left(-\xi^{2}\right) /\{1+\operatorname{erf}(\xi)\} \\
& B=\sqrt{ } \pi \xi\{1+\operatorname{erf}(\xi)\} \exp \left(\xi^{2}\right)
\end{aligned}
$$

The values of $g(B)$ are shown in Table 1. Equation (19a) is the generalization of the potential flow theory (Higbie's penetration theory substituted $x / u_{\infty}$ into the time $t$ ) taking account of the diffusional velocity due to rapid mass transfer. When $B \rightarrow-1$ or $B \rightarrow \infty$, the following asymptotic solutions are easily obtained from Eqs. (19b) and (19c).

$$
\begin{array}{ll}
g(B)=(\pi / 2)^{1 / 2}(1+B)^{-1 / 2} & (B \rightarrow-1) \\
B=\{-\pi \ln 2 g(B)\}^{1 / 2} / g(B) & (B \rightarrow \infty)
\end{array}
$$

Our calculations agreed quite well with Eqs. (20) and (21). Equation (19 a) shows that the effect of transfer number on mass transfer rates is identical for any value of Schmidt number.

When Schmidt number is sufficiently large and $u_{s} \neq 0$, we get the following approximate relation by using the method described in the previous paper ${ }^{15}$.

\begin{tabular}{|c|c|c|c|c|c|}
\hline \multicolumn{6}{|c|}{ Table 1 Values of $g(B)$} \\
\hline$\xi$ & $B$ & $g(B)$ & $\xi$ & $B$ & $g(B)$ \\
\hline-0.0000 & 0.0000 & 1.000 & 0.0000 & 0.000 & 1.0000 \\
\hline-0.0603 & -0.1000 & 1.069 & 0.2169 & 0.500 & 0.7688 \\
\hline-0.1299 & -0.2000 & 1.151 & 0.3578 & 1.000 & 0.6343 \\
\hline-0.2116 & -0.3000 & 1.250 & 0.4608 & 1.500 & 0.5445 \\
\hline-0.3101 & -0.4000 & 1.374 & 0.5411 & 2.000 & 0.4796 \\
\hline-0.4328 & -0.5000 & 1.534 & 0.6618 & 3.000 & 0.3910 \\
\hline-0.5928 & -0.6000 & 1.751 & 0.7506 & 4.000 & 0.3326 \\
\hline-0.8174 & -0.7000 & 2.070 & 0.8204 & 5.000 & 0.2908 \\
\hline-0.9719 & -0.7500 & 2.297 & 0.8776 & 6.000 & 0.2593 \\
\hline-1.175 & -0.8000 & 2.604 & 0.9259 & 7.000 & 0.2345 \\
\hline-1.464 & -0.8500 & 3.053 & 0.9676 & 8.000 & 0.2144 \\
\hline-1.931 & -0.9000 & 3.802 & 1.004 & 9.000 & 0.1978 \\
\hline-2.065 & -0.9100 & 4.022 & 1.037 & 10.00 & 0.1838 \\
\hline-2.223 & -0.9200 & 4.282 & 1.160 & 15.00 & 0.1371 \\
\hline-2.411 & -0.9300 & 4.595 & 1.245 & 20.00 & 0.1104 \\
\hline-2.643 & -0.9400 & 4.983 & 1.362 & 30.00 & 0.08045 \\
\hline-2.932 & -0.9500 & 5.472 & 1.442 & 40.00 & 0.06388 \\
\hline-3.317 & -0.9600 & 6.124 & 1.502 & 50.00 & 0.05325 \\
\hline-3.894 & -0.9700 & 7.116 & 1.551 & 60.00 & 0.04581 \\
\hline-4.301 & -0.9750 & 7.819 & 1.591 & 70.00 & 0.04028 \\
\hline-4.848 & -0.9800 & 8.768 & 1.625 & 80.00 & 0.03601 \\
\hline-5.642 & -0.9850 & 10.15 & 1.655 & 90.00 & 0.03260 \\
\hline-6.964 & -0.9900 & 12.47 & 1.682 & 100.0 & 0.02981 \\
\hline
\end{tabular}

$$
\left(1-w_{s}\right) S h_{x} R e_{x}^{-1 / 2}=(S c / \pi)^{1 / 2}\left(u_{s} / u_{\infty}\right)^{1 / 2} g(B)
$$

Equation (22) shows that the effect of each parameter in Eq. (13) on mass transfer rates is independent of other parameters, and there is no interaction among

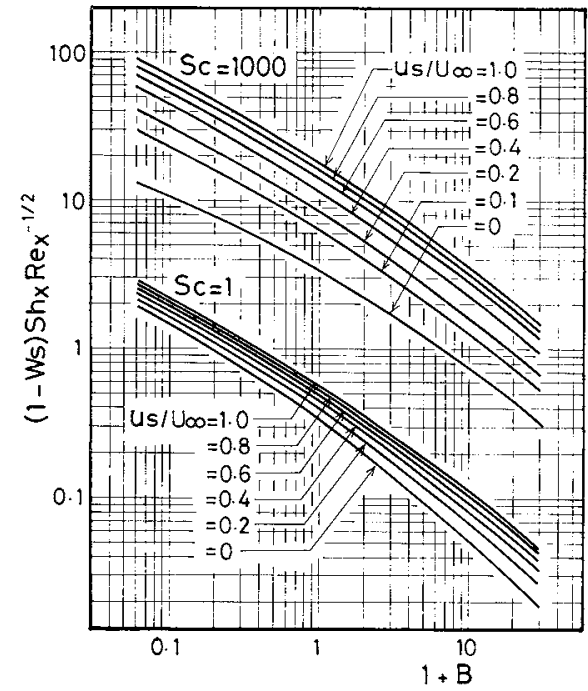

Fig. 7 Effect of transfer number on mass transfer rate

them. The effect of transfer number is identical to that of $u_{s} / u_{\infty}=1$. Our numerical results agreed with Eq. (19a) perfectly. Equation (22) and numerical results were in good agreement for large Schmidt number (see Fig. 7 ; curves corresponding to $u_{s} / u_{\infty}$ are nearly parallel for $S c=1000$ ). The effect of transfer number on mass transfer rates was studied by Evans ${ }^{9}$, Hartnett and Eckert ${ }^{12}$ in the case of low Schmidt number and by Hanna ${ }^{11}$, Merk ${ }^{16)}$ and Stewart ${ }^{21 !}$ in the high Schmidt number region with fixed interface. Our numerical results were also in good agreement with their results.

The independence of the effect of transfer number on mass transfer rates, even for the low Schmidt number region, implies that previous theories of high 


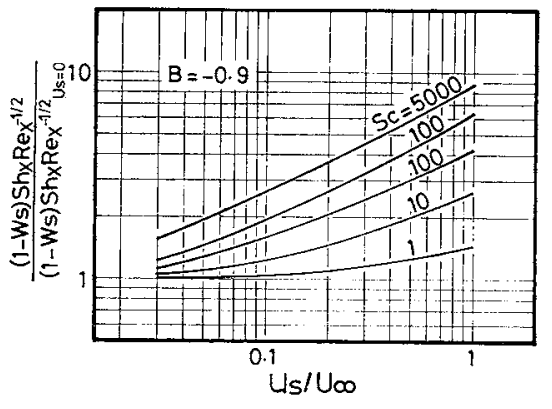

Fig. 8 Effect of interfacial velocity on mass transfer rate

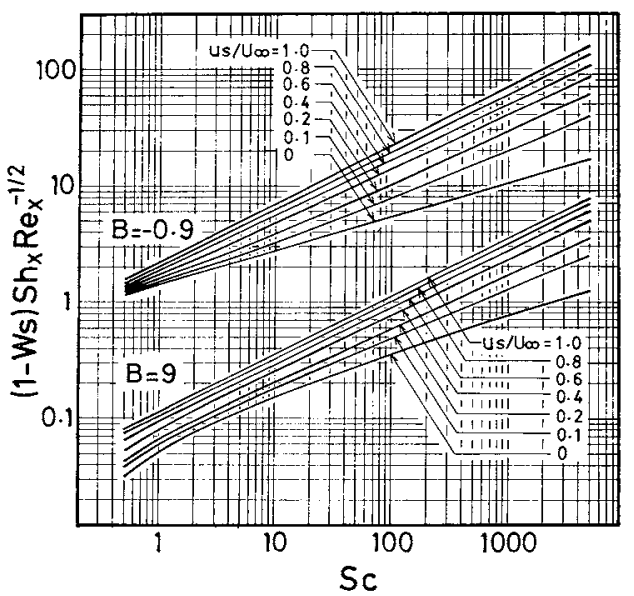

Fig. 9 Effect of Schmidt number on mass transfer rate

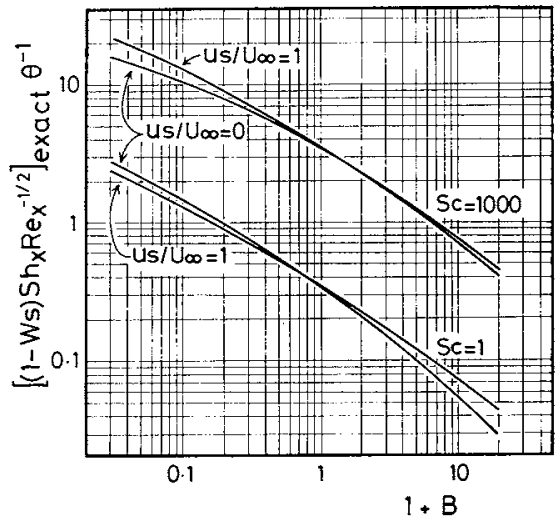

Fig. 10 Comparison of approximate equation and exact solutions

mass flux mass transfer with fixed interface is applicable to high mass flux phenomena with mobile interface. The effect of transfer number on mass transfer rates can be expressed satisfactorily by the correlation predicted from the potential flow theory.

2.3 Effects of interfacial velocity and Schmidt number on mass transfer rates

The independence of the effect of transfer number on mass transfer rates from the interfacial velocity and Schmidt number suggests an interaction between the interfacial velocity and the Schmidt number. The effects of interfacial velocity and Schmidt number on mass transfer rates are shown in Figs. 8 and 9 respectively. The effects of interfacial velocity on mass transfer rates become very important in the case of high Schmidt number, as shown as Fig. 8. In this case we get the following relation from Eq. (22).

$$
\left(1-w_{s}\right) S h_{x} \operatorname{Re}_{x}{ }^{-1 / 2} \propto\left(u_{s} / u_{\infty}\right)^{1 / 2}
$$

On the other hand, the effect of interfacial velocity cannot be expressed by a simple form such as Eq. (21) in the case of low Schmidt number. This effect of increasing mass transfer rates is diminished with decrease of Schmidt number.

The mass transfer rate is proportional to $S c^{1 / 2}$ in the case of $u_{s} / u_{\infty}=1$, as presented in Eq. $(19 \mathrm{a})$. The effect of Schmidt number on mass transfer rate is diminished with decreasing $u_{s} / u_{\infty}$, and the mass transfer rate is in proportion to $S c^{1 / 3}$ in the absence of interfacial velocity, as shown in Fig. 9. Therefore, the effect of Schmidt number on mass transfer rate may be expressed by some value lying between $S c^{1 / 3}$ and $S c^{1 / 2}$ for the case $0<u_{s} / u_{\infty}<1$. For sufficiently large Schmidt number, the effect of Schmidt number on mass transfer rates may be expressed by $S c^{1 / 2}$ even if $u_{s} / u_{\infty}$ has small value, as shown in Eq. (22) and Fig. 9. This effect of interfacial velocity on the index of Schmidt number is very important for the prediction of mass transfer rates.

One of the authors ${ }^{14)}$ presented the following approximate expressions for the interaction between interfacial velocity and Schmidt number in the case of $B \simeq 0$ (low mass flux condition) from the numerical results.

$$
\begin{aligned}
& \left(1-w_{s}\right) S h_{x} R e_{x}^{-1 / 2}=\left\{\left(1-w_{s}\right) S h_{x} R e_{x}^{-1 / 2}\right\}_{u_{s}=0} \theta\left(u_{s} / u_{\infty}, S c\right) \\
& \left\{\left(1-w_{s}\right) S h_{x} R e_{x}^{-1 / 2}\right\}_{u_{s}=0}=0.332 S c^{1 / 3} \\
& \theta=\left\{1+\left(2.88-a S c^{-b}\right)\left(u_{s} / u_{\infty}\right) S c^{1 / 3}\right\}^{1 / 2} \\
& b=3\left(u_{s} / u_{\infty}\right) /\left\{2+12\left(u_{s} / u_{\infty}\right)\right\}
\end{aligned}
$$

$\theta$ is the correction factor for the fixed interface results corresponding to the interfacial velocity and parameter $a$ is the same as given in Eq. (18b). Equation (24a) is applicable for Schmidt number $0.5<S c<5000$ and a wide range of $u_{s} / u_{\infty}$ as well as Eq. (18a), and the error does not exceed $\pm 1 \%$.

Several experiments were made for high mass flux phenomena in the presence of interfacial flow in the low Schmidt number region, and good agreement was obtained with the theories of the fixed interfaces). Clark and $\mathrm{King}^{73}$ obtained good agreement between their experimental results in evaporation of pure volatile liquids and the theory of the potential flow. It may be said that the small effect of interfacial flow due to low Schmidt number was responsible for this agreement. 


\section{4 Approximate analysis}

An asymptotic solution for the mass transfer rates can be obtained for the case of $B \rightarrow-1$ by using the technique presented by Acrivos ${ }^{2}$ as follows:

$$
\left(1-w_{s}\right) S h_{x} R e_{x}^{-1 / 2}=\left[\frac{S c\left\{1+\left(u_{s} / u_{\infty}\right) S c\right\}}{2(1+B)(1+S c)}\right]^{1 / 2}
$$

When $u_{s} / u_{\infty}=1$, Eq. (25) and Eq. (20) are identical. Erickson et al. ${ }^{8,10)}$ analyzed mass transfer toward a moving flat plate within a stationary fluid and gave the following result for $B \rightarrow-1$.

$$
\left(1-w_{s}\right) S h_{\infty} R e_{\infty}^{\prime-1 / 2}=S c /\{2(1+B)(1+S c)\}^{1 / 2}
$$

where

$$
R e_{x}^{\prime}=u_{s} x / \nu
$$

Equation (26a) can be regarded as a special case of $u_{s} / u_{\infty} \gg 1$. Sadek ${ }^{18\rangle}$ verified the good agreement between his experimental results and Eq. (26a).

\section{Approximate Expression for High Mass Flux Mass Transfer with Mobile Interface}

Since most mass transfer coefficients are based on a fixed interface and low mass flux conditions, it is convenient to predict the interfacial flow and high mass flux performance by applying numerical correction factors to the results for the fixed interface and low mass flux conditions. Fortunately, the effect of transfer number on mass transfer rates is highly independent of the other two parameters, $S c$ and $u_{s} / u_{\infty}$, and it may be expressed by the single function $g(B)$, i. e. correction factor for the low mass flux condition. Therefore, the following approximate expression is obtained by combining Eq. (24 a) and $g(B)$.

$$
\left(1-w_{s}\right) S h_{x} R e_{x}^{-1 / 2}=0.332 S c^{1 / 3} \theta\left(u_{s} / u_{\infty}, S c\right) g(B)
$$

A comparison of Eq. (27) and exact solutions is shown in Fig. 10. The curves of Eq. (27) and that of the exact solutions of $u_{s} / u_{\infty}=1$ overlap each other because of accurate correction by $\theta$. The curves corresponding to various $u_{s} / u_{\infty}$ should lie between the curves of $u_{s} / u_{\infty}=0$ and 1 . Equation (27) has sufficient accuracy, as shown in Fig. 10.

\section{Conclusions}

A theoretical study was made of high mass flux mass transfer with mobile interface by using the laminar boundary layer theory. The main results of the work described in this paper are:

1) The interfacial velocity increases mass transfer rates under high mass flux conditions as well as low mass flux conditions. This effect is very important in the high Schmidt number region.

2) The effects of interfacial velocity on mass transfer rates under high mass flux conditions are nearly identical to those under low mass flux conditions. Therefore, the previous theories of high mass flux phenomena with fixed interface are applicable to high mass flux phenomena with mobile interface as long as the increase of mass transfer rates due to interfacial velocity are considered.

It is very important to take account of the interfacial and diffusional velocities for prediction of mass transfer rates, especially in the high Schmidt number region.

\section{Acknowledgment}

This research was supported in part by a grant from the Annual Project organized by Waseda University in 1975 and 1976.

\section{Nomenclature}

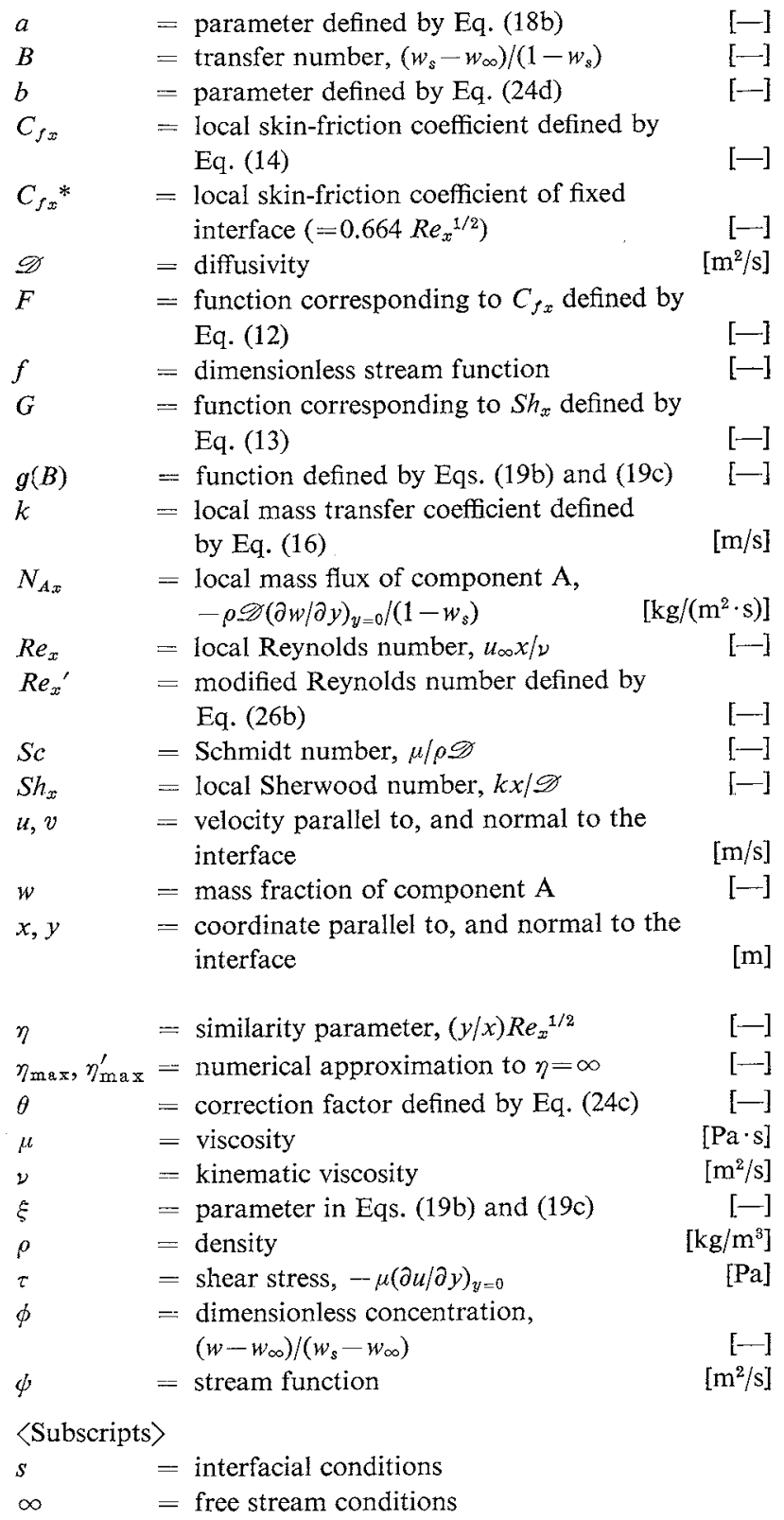

\section{Literature Cited}

1) Acrivos, A.: Chem. Eng. Sci., 9, 242 (1958).

2) idem: AIChE J., 6, 410 (1960).

3) Asano, K.: Kagaku Kōgaku, 28, 538 (1964).

4) Asano, K., Y. Nakano and M. Inaba: J. Chem. Eng. Japan, 
12, $196(1979)$.

5) Bird, R. B., W. E. Stewart and E. N. Lightfoot: "Transport Phenomena", p. 668, Wiley, New York (1960).

6) Cardner, D. V. and J. D. Hellums: Ind. Eng. Chem., Fundam., 6, 376 (1967).

7) Clark, M. W. and C. J. King: AIChE J., 16, 64 (1970).

8) Erickson, L. E., L. T. Fan and V. G. Fox: Ind. Eng. Chem., Fundam., 5, 19 (1966).

9) Evans, H. L.: Int. J. Heat Mass Transfer, 3, 321 (1961).

10) Fox, V. G., L. E. Erickson and L. T. Fan: AIChE J., 14, 726 (1968).

11) Hanna, O. T.: ibid., 9, 704 (1963).

12) Hartnett, J. P. and E. R. G. Eckert: Trans. ASME, Ser. C, 79, 247 (1957).

13) Hirata, A.: Kagaku Kögaku, 37, 551 (1973).

14) Hirata, A., K. Nagasawa and I. Koshijima: Preprint of the 41st Annual Meeting of The Soc. of Chem. Engrs., Japan, Sendai, D 303 (1976).
15) Hirata, A., K. Kira and Y. Suzuki: J. Chem. Eng. Japan, 12, 474 (1979).

16) Merk, H. J.: App. Sci. Res., A-8, 237 (1959).

17) Nienow, A. W.: Brit. Chem. Eng., 12, 1737 (1967).

18) Sadek, S. E.: Ind. Eng. Chem., Fundam., 7, 321 (1968).

19) Shirotsuka, T., A. Hirata and K. Sakai: Kagaku Kögaku, 33, 168 (1969).

20) Shirotsuka, T. and A. Hirata: ibid., 34, 904 (1970).

21) Stewart, W. E.: AIChE J., 8, 421 (1962).

22) idem: ibid., 9, 528 (1963).

23) Stewart, W. E., J. B. Angelo and E. N. Lightfoot: ibid., 16, $771(1970)$.

24) Ueyama, K., J. Hatanaka and K. Ogawa: J. Chem. Eng. Japan, 5, 371 (1972).

(Presented at the 12th Autumn Meeting of The Soc. of Chem. Engrs., Japan, at Okayama, 1978.)

\title{
EFFECT OF OSCILLATORY INSTABILITY ON STABILITY OF TWO-FLUID LAYERS
}

\author{
NobuYuki IMAISHI AND KATSUHIKo FUJINAWA \\ Chemical Research Institute of Non-Aqueous Solutions, \\ Tohoku University, Sendai 980 \\ TEIRIKI TADAKI \\ Department of Chemical Engineering, Tohoku University, \\ Sendai 980
}

\begin{abstract}
Condition for the onset of interfacial turbulence in mass transfer is investigated by a linear stability analysis. In a previous paper, it was revealed that inclusion of the Rayleigh effect as well as the Marangoni effect into analysis alters the stability limit and removes the contradiction between many experimental results and the Sternling and Scriven criteria of stability.

In this paper, the analysis is further extended to include oscillatory instability, which was neglected in the previous analysis.

The results show that oscillatory instability takes an important role in the onset of instabilities, and also that oscillatory instability always takes place when the destabilizing Rayleigh effect and the stabilizing Marangoni effect are competing.

It is suggested that the oscillatory instability of this kind may be correlated to the onset of violent interfacial turbulence or eruption.
\end{abstract}

\section{Introduction}

In a previous paper ${ }^{2)}$, it was revealed that the Rayleigh effect has a profound destabilizing influence upon Marangoni convection caused by interfacial mass transfer in horizontal two-fluid layers confined between two solid walls. In performing the analysis, however, it was assumed without proof that the marginal stability is stationary rather than oscillatory. This as-

Received November 29, 1979. Correspondence concerning this article should be addressed to $\mathrm{N}$. Imaishi. sumption, referred to as the principle of exchange of stabilities, had been justified for the pure Rayleigh convection problem ${ }^{31}$ and the pure Marangoni convection problems ${ }^{1,6)}$ of single-fluid layers. Sternling and Scriven $^{5}$, however, found that both stationary and oscillatory instabilities appear in the pure Marangoni convection problem in two-fluid layers.

In this paper, the same subject as ref. 2) is analysed again, without assuming the exchange of stabilities in order to assess the influence of oscillatory instability on the stability of two-fluid layers subjected to both the Rayleigh and the Marangoni effects in concert. 